\title{
State Space-Based Method for the DOA Estimation by the Forward-Backward Data Matrix Using Small Snapshots
}

\author{
Jianfeng Liu
}

\begin{abstract}
In this presentation, a new low computational burden method for the direction of arrival (DOA) estimation from noisy signal using small snapshots is presented. The approach introduces State Space-based Method (SSM) to represent the received array signal, and uses small snapshots directly to form the Hankel data matrix. Those Hankel data matrices are then utilized to construct forward-backward data matrix that is used to estimate the state space model parameters from which the DOA of the incident signals can be extracted. In contrast to existing methods, such as MUSIC, Root-MUSIC that use the covariance data matrix to estimate the DOA and the sparse representation (SR) based DOA which is obtained by solving the sparsest representation of the snapshots, the SSM algorithm employs forward-backward data matrix formed only using small snapshots and doesn't need additional spatial smoothing method to process coherent signals. Three numerical experiments are employed to compare the performance among the SSM, Root-MUSIC and SR-based method as well as Cramér-Rao bound (CRB). The simulation results demonstrate that when a small number of snapshots, even a single one, are used, the SSM always performs better than the other two method no matter under the circumstance of uncorrelated or correlated signal. The simulation results also show that the computational burden is reduced significantly and the number of antenna elements is saved greatly.
\end{abstract}

Keywords - state space-based method, DOA estimation, small snapshots, array signal, antenna elements, forward-backward data Matrix

\section{INTRODUCTION}

$\mathbf{T}$ There are many algorithms that are used to estimate the direction of arrival (DOA) and much research is going on to enhance their estimate accuracy as well as to reduce their computational burden[1]-[3]. More advanced techniques based on the subspace of the signal such as MUSIC, Root-MUSIC provide the high resolution DOA estimation. MUSIC algorithm [4] estimates the spatial spectrum at all spatial angles. Root-MUSIC[5]-[7]computes the discrete poles estimates along with the corresponding DOA. Much literature provides the performance analysis of these approaches, i.e. [7]-[13]. The existing algorithms such as MUSIC and Root-MUSIC assume that the signals impinging on the array are non-coherent. Under the uncorrelated condition, the covariance matrix satisfies the full rank condition. If the incident signals are correlated with each other, the full rank property of the covariance matrix is not satisfied anymore, those subspace-based algorithms failed

Author is with the Department of Electronic Engineering and Information Science, University of Science and Technology of China, China (e-mail: jfl@mail.ustc.edu.cn). completely. Furthermore, in order to generate a very accurate estimate, the subspace-based algorithms must use large snapshots and more array elements to estimate the covariance matrix which causes the heavy computational burden.

Recently, some new DOA estimation methods based on the sparse signal recovery have been proposed [15]-[16], which are based on the property that the spatial spectrum of the source signals is sparse when the number of signals is limited. In accordance with sparse signal recovery, the sensing matrix is composed of a deterministic overcomplete dictionary which is formed by using the discretization grid of potential DOAs. Thus, the DOA estimation is converted into a problem of recovering a sparse vector with an over-complete dictionary, which can be achieved by solving the convex optimization problem [14], [17]. Compared with the conventional DOA estimation methods, the methods based on the sparse representation (SR) can obtain better estimation precision. However, since the true DOAs will rarely be exactly aligned with the discretization grid, the DOA estimation precision is restricted by the resolution of the grid set. A dense grid is necessary to achieve fine resolution, but too fine grid division will result in large computation time, so the overcomplete dictionary makes the relationship of tradeoff between computation time and estimation accuracy. Although the methods based on the sparse representation (SR) have higher resolution, they suffer from the heavy computational load, and the calculation complexity increases quickly with the number of snapshots. So those kinds of DOA estimation method are not suited for real time applications.

[18]-[20] have developed state space model which is one of the most useful approaches for frequency estimation of signals composed of a sum of exponentials perturbed by noise. The idea of employing state space method for frequency estimation was discussed in [21] in great detail. Their research results show that state space approach performs better than other algorithms in the presence of perturbation. In addition, state space method directly provides the amplitude, phase and the frequencies estimates simultaneously.

By analogy with the harmonic retrieval problem in literature [22], [23], we relate state space model to the received array signal, and this relation is given by the model parameter containing the DOA of the incident signals, then the DOA information can be obtained from the model parameter based on the fact that $e^{j \omega_{k}}=e^{j 2 \pi d / \lambda \cos \left(\theta_{k}\right)}$ where $d, \lambda$ denote the wavelength and the space between the two adjacent array elements respectively, and $\theta_{k}$ denotes the direction of arrival from the $k$ th incident signal, Once $\omega_{k}$ is estimated using state space-based 
method (SSM), The DOA of the $k$ th incident signal can be also obtained .

\section{DATA MODEL}

It is assumed that the narrowband sources are located in the far field of the antenna array and each array element is isotropic omni-directional point sensors radiating in free space. Let us consider a uniformly linear antenna array (ULA) with $M$ of elements into which $K$ signals impinge from the direction $\theta_{k}$. $\theta_{k}$ denotes the direction of arrival of the $k$ th incident signal. Therefore, the $M \times 1$ vector $\boldsymbol{y}(t)=\left[\begin{array}{lll}y_{0}(t) & y_{1}(t) \cdots y_{M-1}(t)\end{array}\right]^{T}$ is the set of voltages measured at the feed point of the antenna elements of the ULA, which is written as

$$
\boldsymbol{y}(t)=\sum_{k=1}^{K} \boldsymbol{a}\left(\theta_{k}\right) s_{k}(t)+\boldsymbol{n}(t)
$$

where $s_{k}(t), k=1 \ldots K$ denotes the $k$ th incident signal coming from the $k$ th direction $\theta_{k}, \boldsymbol{a}\left(\theta_{k}\right)=\left[\begin{array}{ll}a_{0}\left(\theta_{k}\right) & a_{1}\left(\theta_{k}\right) \cdots a_{M-1}\left(\theta_{k}\right)\end{array}\right]^{T}$ denotes the $M \times 1$ steering vector at $\theta_{k},[]^{T}$ denotes the matrix transpose, and $\boldsymbol{n}(t)=\left[n_{0}(t) n_{1}(t) \cdots n_{M-1}(t)\right]^{T}$ denotes the $M \times 1$ vector of noise.

Eq. (1) can be written as following:

$$
\boldsymbol{y}(t)=\left[\begin{array}{c}
y_{0}(t) \\
y_{1}(t) \\
\vdots \\
y_{M-1}(t)
\end{array}\right]=\left[\begin{array}{cccc}
a_{0}\left(\theta_{1}\right) & a_{0}\left(\theta_{2}\right) & \cdots & a_{0}\left(\theta_{K}\right) \\
a_{1}\left(\theta_{1}\right) & a_{1}\left(\theta_{2}\right) & \cdots & a_{1}\left(\theta_{K}\right) \\
\vdots & \vdots & & \vdots \\
a_{M-1}\left(\theta_{1}\right) & a_{M-1}\left(\theta_{2}\right) & \cdots & a_{M-1}\left(\theta_{K}\right)
\end{array}\right]\left[\begin{array}{c}
s_{1}(t) \\
s_{2}(t) \\
\vdots \\
s_{K}(t)
\end{array}\right]+\left[\begin{array}{c}
n_{0}(t) \\
n_{1}(t) \\
\vdots \\
n_{M-1}(t)
\end{array}\right]
$$

In the absence of noise, the signal received by the mth element is expressed as

$$
y_{m}(t)=\sum_{k=1}^{K} a_{m}\left(\theta_{k}\right) s_{k}(t), m=0 \ldots . M-1
$$

where $a_{m}\left(\theta_{k}\right), m=0 \ldots . M-1$ denote the mth component of the steering vector $\boldsymbol{a}\left(\theta_{k}\right)$. Therefore, we can write the sampled signal as

$$
y_{m}[n]=\sum_{k=1}^{K} a_{m}\left(\theta_{k}\right) s_{k}[n], m=0 \ldots . M-1, n=1 \ldots . N
$$

The above Eq. (4) can be further written as

$$
y_{m}[n]=\sum_{k=1}^{K} s_{k}[n] e^{j \omega_{k} m}, m=0 \ldots . M-1, n=1 \ldots . . N
$$

where $e^{j \omega_{k} m}=a_{m}\left(\theta_{k}\right), \omega_{k}=2 \pi d / \lambda \cos \left(\theta_{k}\right)$ and $d, \lambda$ denote the wavelength and the space between the two adjacent array elements respectively.

\section{STATE SPACE REPRESENTATION FOR THE ARRAY SIGNAL}

In this section, we employ a special method to model the array signals by use of the state-space method and establish low rank Hankel data matrix using the nth snapshot received by antenna array. Generally, the input-output relationship for the general autoregressive moving average (ARMA) model is given by the following difference equation:

$$
u[m]=\sum_{k=1}^{K} a_{k} u[m-k]+\sum_{k=0}^{q} b_{k} v[m-k]
$$

where $v[n], u[n]$ denote the input and the output respectively. In the absence of noise, the signal received by the $m$ th element at the nth snapshots is rewritten as

$$
y_{m}[n]=\sum_{k=1}^{K} s_{k}[n] e^{j \omega_{k} m}, m=0 . . . M-1, n=1 \ldots . N
$$

The above equation can be considered to be a sum of $K$ complex exponentials at the nth snapshot. Such a signal is assumed to be the output of a self-generating system (zero-input oscillators). It means that a special ARMA model is employed whose poles are all on the unit circle and input powers are all 0 's, which is expressed as

$$
u[m]=\sum_{k=1}^{K} a_{k} u[m-k]
$$

Substituting Eq.(7) into Eq.(8), then it can be written as

$$
y_{m}[n]=\sum_{k=1}^{K} a_{k} y_{m-k}[n]
$$

Here we introduce the state-space method to modeling the array signal. The state-space representation for the signal received by the $m$ th element at the nth snapshot is defined as

$$
\begin{aligned}
& \boldsymbol{X}_{m+1}[n]=\boldsymbol{F} \boldsymbol{X}_{m}[n] \\
& y_{m}[n]=\boldsymbol{h} \boldsymbol{X}_{m}[n]
\end{aligned}
$$

where $\boldsymbol{X}_{m}[n]$ is a $K \times 1$ state vector at the nth snapshot and $\boldsymbol{F}$ is constant matrix of size $K \times K$.The literature[18],[20] has proved that the matrix $\boldsymbol{F}$ provide the frequencies information. So we can obtain the DOA of the incident signals from the matrix $\boldsymbol{F}$.The special case is

$$
\boldsymbol{F}=\left[\begin{array}{rrr}
e^{j \omega_{1}} & & \\
& e^{j \omega_{2}} & \\
& \ddots & \\
& & e^{j \omega_{K}}
\end{array}\right]
$$

where $\omega_{k}=2 \pi d / \lambda \cos \left(\theta_{k}\right)$.This is an interesting and useful result, because the parameter matrix $\boldsymbol{F}$ hides the DOA information. According to the above analysis, the DOA estimation problem is then converted to solve the parameter matrix $\boldsymbol{F}$. Thus our study focuses on the estimation of $\boldsymbol{F}$ from the incident signals received by the $\mathrm{mth}$ element at the $\mathrm{nth}$ snapshot. From the state-space equations Eq. (10), after some manipulations, we have

$$
y_{m}[n]=\boldsymbol{h F}^{m} \boldsymbol{X}_{0}[n]
$$

Then the Hankel matrix directly formed from the array data signal at the nth snapshots is written as

$$
\boldsymbol{Y}[n]=\left[\begin{array}{ccccc}
y_{0}[n] & y_{1}[n] & y_{2}[n] & \cdots & y_{M-L}[n] \\
y_{1}[n] & y_{2}[n] & y_{3}[n] & \cdots & y_{M-L+1}[n] \\
y_{2}[n] & y_{3}[n] & y_{4}[n] & \cdots & y_{M-L+2}[n] \\
\vdots & \vdots & \vdots & \vdots \\
y_{L-1}[n] & y_{L}[n] & y_{L+1}[n] & \cdots & y_{M-1}[n]
\end{array}\right] \quad K<L<M
$$

Combining Eq. (12) with Eq. (13), Eq. (13) can be further factorized as follows [18]-[20]: 


$$
\boldsymbol{Y}[n]=\left[\begin{array}{l}
\boldsymbol{h} \\
\boldsymbol{h} \boldsymbol{F}^{1} \\
\boldsymbol{h} \boldsymbol{F}^{2} \\
\vdots \\
\boldsymbol{h} \boldsymbol{F}^{L-1}
\end{array}\right]\left[\boldsymbol{X}_{0}[n] \boldsymbol{F}_{0}[n] \boldsymbol{F}^{2} \boldsymbol{X}_{0}[n] \cdots \boldsymbol{F}^{M-L} \boldsymbol{X}_{0}[n]\right]=\Theta \Phi
$$

where $\left[\boldsymbol{h} \boldsymbol{h} \boldsymbol{F}^{1} \boldsymbol{h} \boldsymbol{F}^{2} \cdots \boldsymbol{h} \boldsymbol{F}^{L-1}\right]^{T}=\Theta \quad$ (15), in which []$^{T}$ denotes the matrix transpose. Since the parameter $\boldsymbol{F}$ contains the DOA information of the incident signals, here we consider the estimation of the parameter $\boldsymbol{F}$ from the factor $\Theta$.

First, $\Theta_{1}, \Theta_{2}$ are defined respectively as following:

$$
\begin{aligned}
& \Theta_{1}=\left[\boldsymbol{h} \boldsymbol{h} \boldsymbol{F}^{1} \boldsymbol{h} \boldsymbol{F}^{2} \cdots \boldsymbol{h} \boldsymbol{F}^{L-2}\right]^{T} \\
& \Theta_{2}=\left[\boldsymbol{h} \boldsymbol{F}^{1} \boldsymbol{h} \boldsymbol{F}^{2} \cdots \boldsymbol{h} \boldsymbol{F}^{L-1}\right]^{T}
\end{aligned}
$$

where []$^{T}$ denotes the matrix transpose. From Eq. (16), we can easily obtain the following equation:

$$
\Theta_{1} \boldsymbol{F}=\Theta_{2} \text {, that is } \boldsymbol{F}=\Theta_{1}^{\dagger} \Theta_{2}
$$

where ${ }^{(.)^{\dagger}}$ denotes the Moore-Penrose pseudo-inverse of matrix. Once the matrix $\boldsymbol{F}$ is computed, the parameter $e^{j \omega_{k}}, k=1 \ldots K$ corresponding to the DOA of the incident signals can be obtai ned by solving the following eigenvalue decomposition.

$$
\boldsymbol{F}=\boldsymbol{U}\left[\begin{array}{cccc}
e^{j \omega_{1}} & & & \\
& e^{j \omega_{2}} & & \\
& & \ddots & \\
& & e^{j \omega_{K}}
\end{array}\right] \boldsymbol{U}^{H}=\sum_{k=1}^{K} \boldsymbol{u}_{k} \boldsymbol{u}_{k}^{H} e^{j \omega_{k}}
$$

where $\boldsymbol{U}=\left[\begin{array}{llll}\boldsymbol{u}_{1} & \boldsymbol{u}_{2} & \cdots & \boldsymbol{u}_{K}\end{array}\right]$.Then the DOA can be computed as follows:

$$
\theta_{k}=\cos ^{-1}\left\{\frac{\Im\left[\ln \left(e^{j \omega_{k}}\right)\right] \lambda}{2 d \pi}\right\}, k=1 \ldots K
$$

where $\mathfrak{I}$ denotes the imaginary part. It's worth noting that the methods for estimating the number of incident signals $K$ are well documented in the literature[23], and will not be discussed here. In this paper we assume that the number of signals $K$ is given.

\section{MULTIPLE SNAPSHOTS FORWARD-BACKWARD DATA MATRIX STRUCTURE}

In order to further improve the DOA estimate accuracy, the multiple snapshots are used in this paper. In the case of multiple snapshots, the data matrix is constructed using each snapshot. It is assumed that there are $N$ snapshots. For a single snapshot, the Hankel data matrix $Y[i]$ is constructed at the $i$ th snapshot as follows:

$$
\boldsymbol{Y}[i]=\left[\begin{array}{ccccc}
y_{0}[i] & y_{1}[i] & y_{2}[i] & \cdots & y_{M-L}[i] \\
y_{1}[i] & y_{2}[i] & y_{3}[i] & \cdots & y_{M-L+1}[i] \\
y_{2}[i] & y_{3}[i] & y_{4}[i] & \cdots & y_{M-L+2}[i] \\
\vdots & \vdots & \vdots & \vdots \\
y_{L-1}[i] & y_{L}[i] & y_{L+1}[i] & \cdots & y_{M-1}[i]
\end{array}\right], i=1 \ldots N, K<L<M
$$

Then $\boldsymbol{Y}$ [1] $\boldsymbol{Y}[2] \ldots \boldsymbol{Y}[N]$ are appended side by side to construct multiple snapshots forward data matrix, which is written as

$$
\boldsymbol{Y}^{f}=\left[\begin{array}{ll}
\boldsymbol{Y}[1] & \boldsymbol{Y}[2] \cdots \boldsymbol{Y}[N]]
\end{array}\right.
$$

Eq.(13) is a special case of the matrix $\boldsymbol{Y}^{f}$ with $i=n$. According to the same method as mentioned above, the multiple snapshots backward structure is expressed as

$$
\boldsymbol{Y}^{b}=[\boldsymbol{Z}[1] \boldsymbol{Z}[2] \ldots \boldsymbol{Z}[N]]
$$

Where

$$
Z[k]=\left[\begin{array}{ccc}
y_{M-1}^{*}[N-k+1] & y_{M-2}^{*}[N-k+1] & \cdots y_{L-1}^{*}[N-k+1] \\
y_{M-2}^{*}[N-k+1] & y_{M-3}^{*}[N-k+1] & \cdots y_{L-2}^{*}[N-k+1] \\
y_{M-3}^{*}[N-k+1] & y_{M-4}^{*}[N-k+1] & \cdots y_{L-3}^{*}[N-k+1] \\
\vdots & \vdots & \vdots \\
y_{M-L}^{*}[N-k+1] & y_{M-L-1}^{*}[N-k+1] & \cdots y_{0}^{*}[N-k+1]
\end{array}\right], k=1 \ldots N
$$

where (.) ${ }^{*}$ denotes the complex conjugation. Hence the multiple snapshots forward-backward data matrix is written as

$$
\boldsymbol{Y}^{f b}=\left[\begin{array}{ll}
\boldsymbol{Y}^{f} & \boldsymbol{Y}^{b}
\end{array}\right]
$$

It can be seen that the data processing approach presented in section 2 and 3 is also applicable to the multiple snapshots forward-backward data matrix.

\section{EXTRACTING THE DOA FROM THE FORWARD-BACKWARD DATA MATRIX IN THE PRESENCE OF NOISE}

The above analysis is based on the fact that the received array signals are free-noise. If the received array signals are perturbed by the noise, the forward-backward data matrix $\boldsymbol{Y}^{f b}$ can be expressed as $\boldsymbol{Y}^{f b}=\hat{\boldsymbol{Y}}^{f b}+\boldsymbol{n}$, which loses the low-rank property, where $\boldsymbol{n}$ denotes the noise matrix and $\hat{\boldsymbol{Y}}^{f b}$ denotes the forward-backward data matrix without noise. So approximate factorization method must be employed, here we use singular value decomposition (SVD). The idea is to decompose the forward-backward data matrix $\boldsymbol{Y}^{f b}$ into the signal and noise subspaces, and keep the signal subspace. Note that we keep the signal subspace and not the noise subspace, which gets used in MUSIC subspace methods. Therefore $\boldsymbol{Y}^{f b}=\hat{\boldsymbol{Y}}^{f b}+\boldsymbol{n}$ can be decomposed as

$$
\begin{aligned}
& \boldsymbol{Y}^{f b}=\hat{\boldsymbol{Y}}^{f b}+\boldsymbol{n}=\left[\begin{array}{ll}
\boldsymbol{U}_{1} & \boldsymbol{U}_{2}
\end{array}\right]\left[\begin{array}{ll}
\Sigma_{1} & \\
& \Sigma_{2}
\end{array}\right]\left[\begin{array}{l}
\boldsymbol{V}_{1}^{H} \\
\boldsymbol{V}_{2}^{H}
\end{array}\right] \\
& =\overbrace{\underbrace{\boldsymbol{U}_{1} \sum_{1} \boldsymbol{V}_{1}^{H}}_{\text {free-noise data }}}^{\hat{\mathbf{r}}^{\text {fb }}}+\overbrace{\underbrace{\boldsymbol{U}_{2} \sum_{2} \boldsymbol{V}_{2}^{H}}_{\text {noise }}}^{n}=\Theta \Phi
\end{aligned}
$$

where $\Sigma_{1}$ denotes the diagonal matrix composed of the dominant singular values, $\boldsymbol{U}_{1}, \boldsymbol{V}_{1}$ denote the left and right singular vector respectively corresponding to the dominant singular values, and $\sum_{2}$ denotes the diagonal matrix composed of the non-dominant singular values, $\boldsymbol{U}_{2}, \boldsymbol{V}_{2}$ denote the left and right singular vector respectively corresponding to the non-dominant singular values.

Thus the pre-filtered noisy forward-backward data matrix processed by SVD is shown as [18]

$$
\hat{\boldsymbol{Y}}^{f b}=\boldsymbol{U}_{1} \sum_{1} \boldsymbol{V}_{1}^{H}=\boldsymbol{U}_{1} \sum_{1}^{1 / 2} \sum_{1}^{1 / 2} \boldsymbol{V}_{1}^{H}=\hat{\Theta} \hat{\Phi}
$$


where $(.)^{H}$ denotes the complex conjugate transpose. Then $\hat{\Theta}=U_{1} \sum_{1}^{1 / 2}$, combining Eq. (15), Eq. (16) with Eq. (17) to obtain $\hat{\Theta}_{1}$ and $\hat{\Theta}_{2}$, thus the estimate of the parameter matrix $\hat{\boldsymbol{F}}$ as follows:

$$
\hat{\boldsymbol{F}}=\hat{\Theta}_{1}^{\dagger} \hat{\Theta}_{2}
$$

where $(.)^{\dagger}$ denotes the Moore-Penrose pseudo-inverse of the ma trix.Thus the DOA estimates are obtained using eigenvalue dec omposition for the parameter matrix $\hat{\boldsymbol{F}}$, i.e.

$$
\begin{gathered}
\hat{\boldsymbol{F}}=\sum_{k=1}^{K} \boldsymbol{u}_{k} \boldsymbol{u}_{k}^{H} e^{j \omega_{k}} \\
\text { then } \hat{\theta}_{k}=\cos ^{-1}\left\{\frac{\mathfrak{I}\left[\ln \left(e^{j \omega_{k}}\right)\right] \lambda}{2 d \pi}\right\}, k=1 \ldots K
\end{gathered}
$$

where $\mathfrak{I}$ denotes the imaginary part. It is clearly seen from the above analysis that by use of SVD, the parameter $\hat{\boldsymbol{F}}$ is robust to perturbation, so the DOA estimate $\hat{\theta}_{k}$ is also insensitive to the noise.

The DOA estimation procedure using the SSM is organized in the following steps:

Step 1: Employing the received array signal $y_{m}[n], n=1 \ldots N$ to form the forward-backward data matrix

$$
\boldsymbol{Y}^{f b}=\left[\begin{array}{ll}
\boldsymbol{Y}^{f} & \boldsymbol{Y}^{b}
\end{array}\right]
$$

Step2: Performing SVD for $\boldsymbol{Y}^{f b}$ to suppress noise perturbation

$$
\begin{aligned}
& \boldsymbol{Y}^{f b}=\left[\begin{array}{ll}
\boldsymbol{U}_{1} & \boldsymbol{U}_{2}
\end{array}\right]\left[\begin{array}{ll}
\sum_{1} & \\
& \Sigma_{2}
\end{array}\right]\left[\begin{array}{l}
\boldsymbol{V}_{1}^{H} \\
\boldsymbol{V}_{2}^{H}
\end{array}\right] \\
& \hat{\boldsymbol{Y}}^{f b}=\boldsymbol{U}_{1} \sum_{1} \boldsymbol{V}_{1}^{H}
\end{aligned}
$$

Step3: Factorizing the pre-processed $\hat{\boldsymbol{Y}}^{f b}$ to obtain $\hat{\Theta}$, i.e.:

$$
\hat{\Theta}=U_{1} \sum_{1}^{1 / 2}
$$

Step4: Computing the model parameter $\boldsymbol{F}$ using Eq. (15), Eq. (16) and Eq. (17)

$$
\hat{\boldsymbol{F}}=\hat{\Theta}_{1}^{\dagger} \hat{\Theta}_{2}
$$

Step5: Performing eigenvalue decomposition for the model parameter $\boldsymbol{F}$ to obtain $e^{j \omega_{k}}$, i.e.

$$
\hat{\boldsymbol{F}}=\sum_{k=1}^{K} e^{j \omega_{k}} \boldsymbol{u}_{k} \boldsymbol{u}_{k}^{H}
$$

Step6: The DOA $\hat{\theta}_{k}$ corresponding to the $k$ th signal can be computed as following:

$$
\hat{\theta}_{k}=\cos ^{-1}\left\{\frac{\mathfrak{I}\left[\ln \left(e^{j \omega_{k}}\right)\right] \lambda}{2 d \pi}\right\}, k=1 \ldots K
$$

where $\mathfrak{I}$ denotes the imaginary part .

\section{PERFORMANCE VIA COMPUTER SIMULATION}

To examine the performance of SSM method in comparison to root-MUSIC and SR method, as well as the the Cramer-Rao lower bound (CRLB), we perform three numerical experiments. The simulations are performed using MATLAB8 running on an Intel Core i5, 3.2 GHz processor with $4 \mathrm{~GB}$ of memory, under Window7. In our study, the uniform linear array (ULA) composed of the omni-directional isotropic point sensors is employed. The distance of adjacent sensor of the ULA is half a wavelength. The performance of the DOA estimation are measured by the root mean square error (RMSE) which is defined as

$$
R M S E=\sqrt{\frac{1}{N} \sum_{n=1}^{N}\left(\hat{\theta}_{k n}-\theta_{k}\right)^{2}}
$$

where $\theta_{k}$ is the desired DOA of the $k$ th incident signal and $\hat{\theta}_{k n}$ is its estimate at the nth trial.

In estimation theory and statistics, the CRLB expresses a lower bound on the variance of estimators. So comparison to the performance of DOA corresponding to the CRLB should be of interest. The bound states that the variance of estimator is at least as high as the inverse of the Fisher information. Defining the Fisher Information Matrix $I(\boldsymbol{\theta})$ whose elements are given as[25][26]

$$
I\left(\theta_{i, j}\right)=-\boldsymbol{E}\left\{\frac{\partial^{2} \ln f(X ; \boldsymbol{\theta})}{\partial \theta_{i} \partial \theta_{j}}\right\} i, j=1,2 \ldots K
$$

Therefore, the CRLB on the variance of $\hat{\theta}$ is given by

$$
C R L B_{\hat{\theta}}=[I(\boldsymbol{\theta})]^{-1}
$$

where $\boldsymbol{E}$ denotes the expectation operator, $\hat{\boldsymbol{\theta}}$ is the estimate of $\boldsymbol{\theta}$, and $f(X, \boldsymbol{\theta})$ is the probability density function conditioned to an unknown parameter $\theta$.

Then the CRLB for the DOA of the $k$ th incident signal can be written as

$$
\operatorname{var}\left(\hat{\theta}_{k}\right) \geq C R L B_{\hat{\theta}_{k k}}
$$

where $\theta_{k}$ is the desired DOA of the $k$ th incident signal. In the following examples, the variance of the DOA estimate with SSM, Root-MUSIC and SR based methods and the corresponding CRLB versus signal-to-noise ratio (SNR) will be shown.

Experiment 1: In this experiment, the noisy signal model is formulated from Eq. (1). Noise is treated as Gaussian white noise which is assumed to be zero mean, complex Gaussian random processes that are statistically independent of each other. It is assumed that the two uncorrelated equal-power signals are impinging on the array which are coming from $84^{\circ}$ and $98^{\circ}$ respectively. $L$ is chosen to be floor $(2 M / 3)$. The CRLB of the DOA is calculated simultaneously to provide a benchmark of estimation performance[25],[26]. In our simulations, the potential DOAs grid division which is employed as the over-complete representation ranges from 0 to 180 with 0.1 spacing for the sparse representation(SR) based method, and the Orthogonal Matching Pursuit (OMP) [27]algorithm is used to perform the signal reconstruction and DOA estimations. The performances of the state space-based Method (SSM), the Root-MUSIC algorithm, sparse representation (SR) based DOA method and the CRLB are compared under the following five scenarios.

In the first scenario, the performance of the SSM is compared with that of Root-MUSIC and the CRLB. There are 7 antenna elements, the SNR changes from $0 \mathrm{~dB}$ to $20 \mathrm{~dB}$ for a single snapshot, 5 snapshots, 10 snapshots and 30 snapshots respectively. In the second scenario, there are also 7 antenna elements, 30 snapshots are used for the SSM and 100,800 snapshots are used for Root-MUSIC respectively, and the SNR changes from $0 \mathrm{~dB}$ to $20 \mathrm{~dB}$. In the third scenario, we compare the performance of the SSM with that of the SR based method 
and the CRLB. There are 7 antenna elements, and the SNR changes from $0 \mathrm{~dB}$ to $20 \mathrm{~dB}$ for a single snapshot, 20 snapshots and 30 snapshots respectively. In the forth scenario, the performance of the SSM is compared with that of SR based method and the Root-MUSIC. The number of snapshots increases from 1 to 100 , there are also 7 antenna elements, the SNR is fixed at $15 \mathrm{~dB}$. In the fifth scenario, the SNR is chosen to be $15 \mathrm{~dB}, 20$ snapshots are used and the number of antenna elements ranges from 7 to 20.1000 independent simulations are conducted each simulation, the results are plotted in Figure 1, Figure 2, Figure 3, Figure 4.

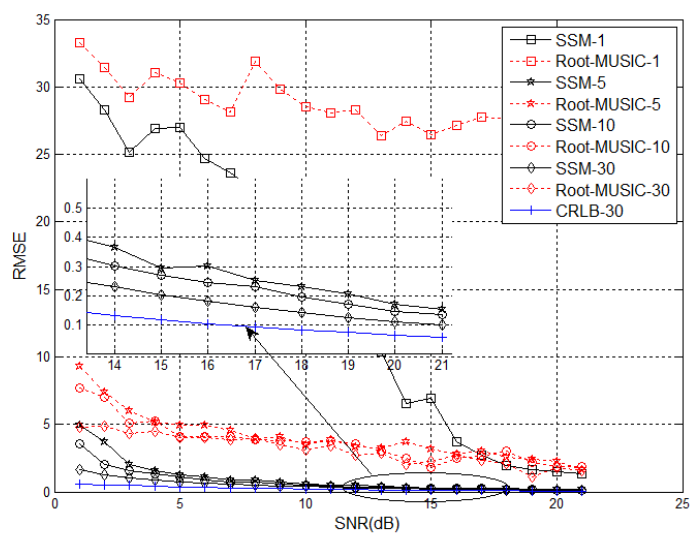

(a)

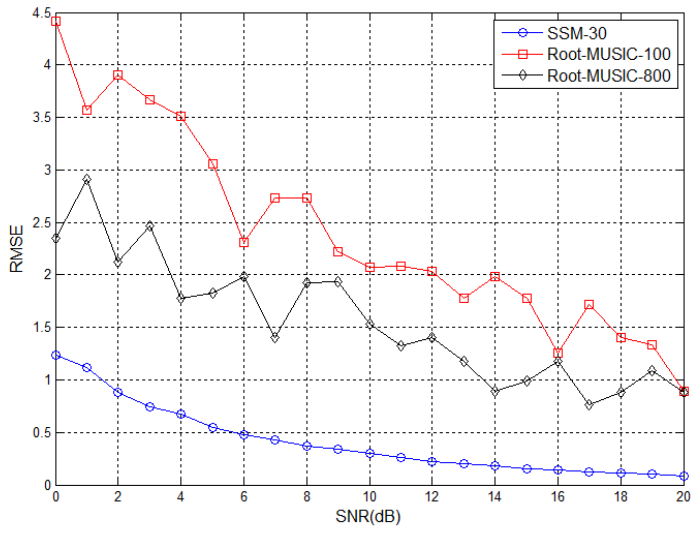

(b)

Fig.1.The RMSE of the DOA versus SNR

As can be seen from Fig. 1(a), the SSM method has a lower RMSE than the Root-MUSIC algorithm under the same number of snapshots, and it follows the CRLB more closely than the Root-MUSIC. Although the estimate accuracy of the Root-MUSIC is improved as the number of snapshots increase, its performance with 100 and 800snapshots is much worse than that of the SSM with 30 snapshots(as shown in Fig.1(b)). Figure 1(a) also shows that the SSM has the same performance at a single snapshot as the Root-MUSIC at 30 snapshots after $20 \mathrm{~dB}$ SNR, and the SSM has a similar performance when using 5, 10 and 30 snapshots after $20 \mathrm{~dB}$ SNR. The simulation results demonstrate that for the SSM, using small snapshots can obtain a very good estimate, and its estimate accuracy outperforms the Root-MUSIC.

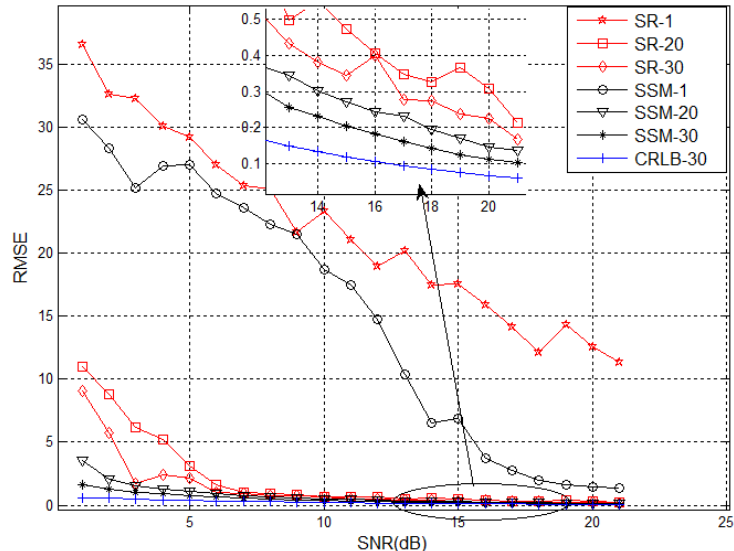

Fig. 2.The RMSE of the DOA versus SNR

Fig. 2 shows that the SSM perform better than the SR based method, and it also follows the CRLB more closely than the SR based method. The simulation results show the effectivity of the SSM under the case of the small number of snapshots compared with the SR based method. The reason is that the forward-backward matrix is employed for the SSM, so under the condition of the same number of snapshots, the SSM method has larger amount of information than SR based method.

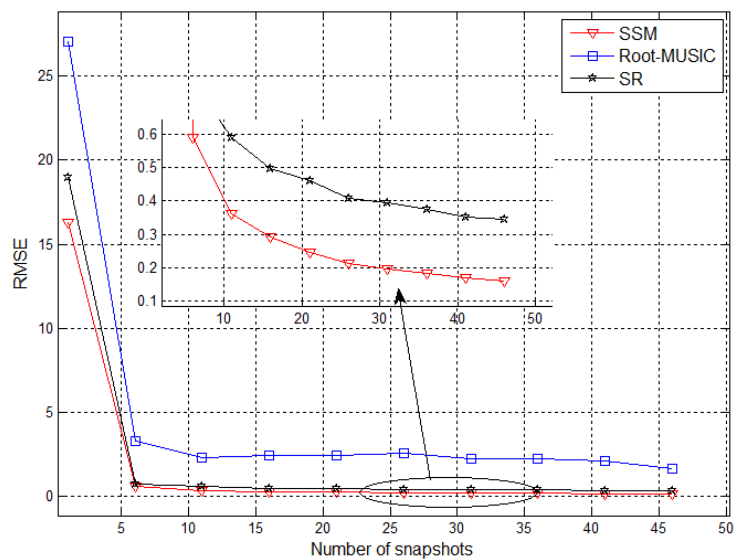

Fig.3. The RMSE of the DOA versus the number of snapshots

Fig. 3 shows that the estimate accuracy of the SSM is the best among all with the fewer number of snapshots when SNR is $15 \mathrm{~dB}$ and 7 antenna elements are used, and the estimation performance of the SR based method lies between the SSM and the Root-MUSIC. Although the SR based method has much lower RMSE than the Root-MUSIC, it is still worse than the SSM. Simulation results validate the effectiveness of the SSM method and illustrate that it has higher estimation precision with the fewer number of snapshots. 


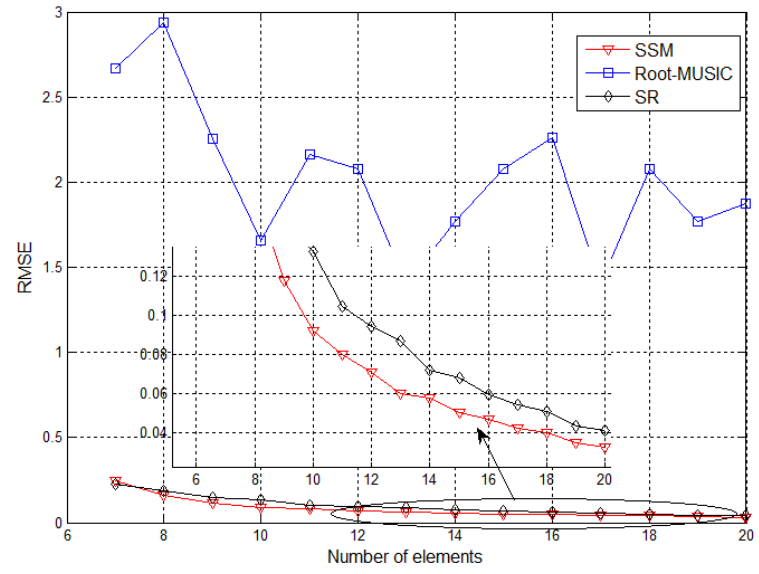

Fig.4. The RMSE of the DOA versus the number of antenna elements

Fig.4 plots curves of RMSE versus the number of antenna elements. As shown in Figure 4, the SSM can obtain better performance than the other two methods with fewer antenna elements under the same condition, so the SSM can save the number of antenna elements and need a smaller aperture than the Root-MUSIC and SR based method.

Experiment 2: In this experiment, we examine the performance of the SSM in comparison to the Root-MUSIC, SR based method and CRLB under the case of the two equal-power correlated signals with a correlation coefficient of 0.99 . This example has the same parameters setting as the example 1 in the first, the second scenario and the third scenario except that the two signals impinging into the array are correlated. The simulation results are shown in Figure 5, Figure 6 and Figure 7.

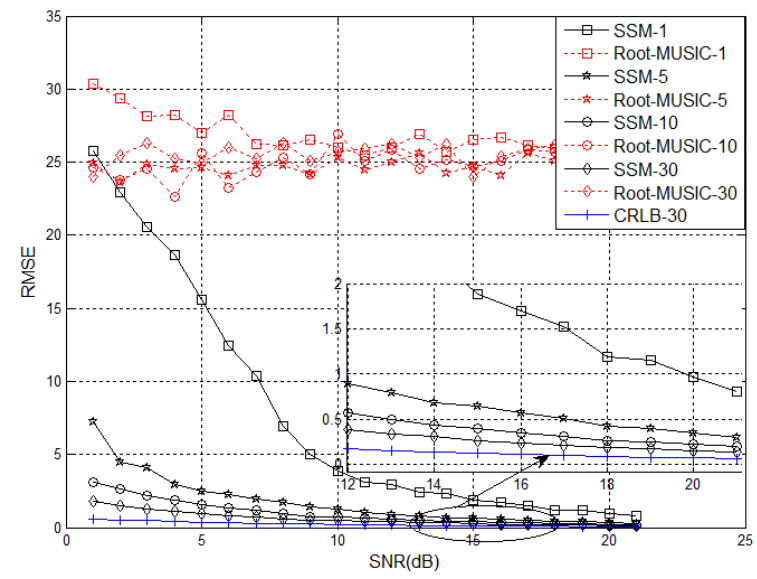

Fig.5. The RMSE of the DOA versus SNR

As shown in Fig. 5, the Root-MUSIC algorithm failed totally, but the SSM demonstrates the perfect performance even at a single snapshot after 20dB SNR. The simulation result shows that the SSM has a superior ability to the Root-MUSIC algorithm using the small number of snapshots under the correlated signal case. For the reason that the covariance matrix of the Root-MUSIC loses the full rank property, it needs some additional spatial smoothing techniques so that it can work for the correlated signal case.

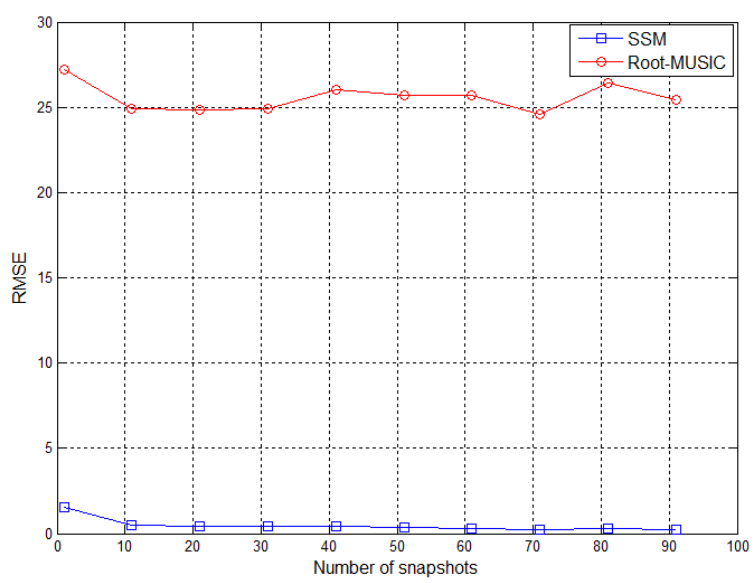

Fig.6. The RMSE of the DOA versus the number of snapshots

Fig. 6 plots curves of RMSE versus the number of snapshots and shows that the Root-MUSIC doesn't work anyway even at 100 snapshots, but the SSM algorithm still work well even at a single snapshot, and no accuracy improvement is observed by further increasing the number of snapshots. The simulation result demonstrates that increasing the number of the snapshots doesn't improve the performance of the Root-MUSIC when the two incident signals are correlated, whereas the SSM performs perfect in the conditions of small snapshots without additional spatial smoothing techniques.

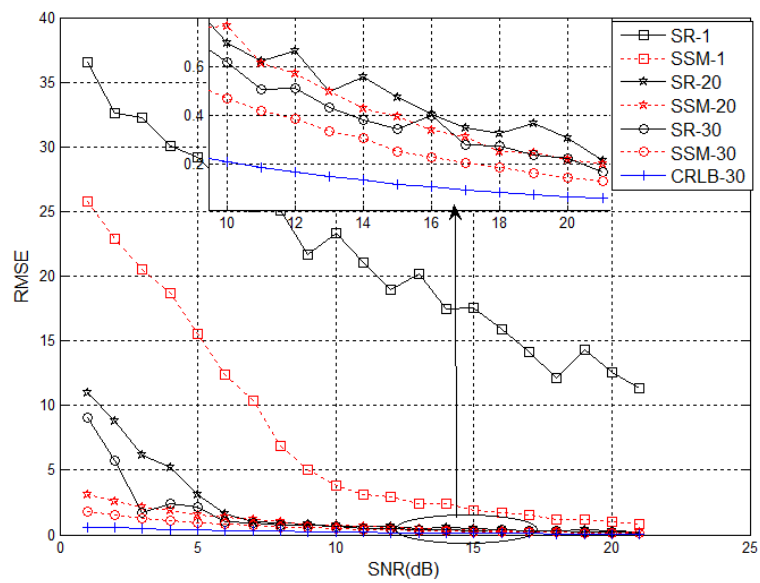

Fig. 7. The RMSE of the DOA versus SNR

Fig. 7 shows that in contrast with Root-MUSIC (as shown in Fig. 5), the SR based method can obtain better estimation performance without any decorrelation preprocessing under the condition of the correlated signals, and the estimation accuracy can be improved by further increasing the number of snapshots, but the RMSE of SR based method is still higher than that of SSM. Also, the SSM follows the CRLB more closely than the SR based method.

Experiment 3: In this experiment, the computational load is examined. The CPU processing time is used as the evaluative criteria. The parameter setting is similar to the example 1(the computation time is similar when sources are correlated). The computational load of the SSM, the Root-MUSIC and SR based 
algorithm is compared under the two scenarios. In the first case, 20 snapshots is used, the SNR is fixed at $20 \mathrm{~dB}$, the number of array elements changes from 7 to 20 . In the second case, 7 elements array are employed, SNR is still $20 \mathrm{~dB}$, the number of snapshots increases from 10 to 100.500 independent simulations are conducted. The simulation results are plotted in Figure.8 and Figure 9, which plot curves of CPU processing time versus the number of elements and the number of snapshots, respectively.

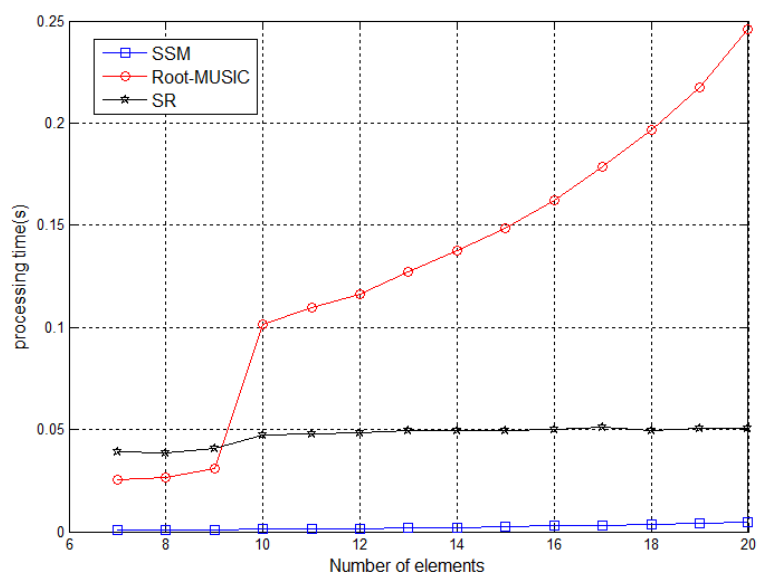

Fig.8. The processing time versus the number of elements

As can be seen from Fig. 8, the SSM is fastest among all and the CPU processing time of the SSM and SR based method keeps unchangeable nearly as the number of array elements increases, however the processing time of the Root-MUSIC increases greatly. The result shows that the increase of the array elements accelerates the computational load of the Root-MUSIC, but the SSM is barely affected by the number of array elements.

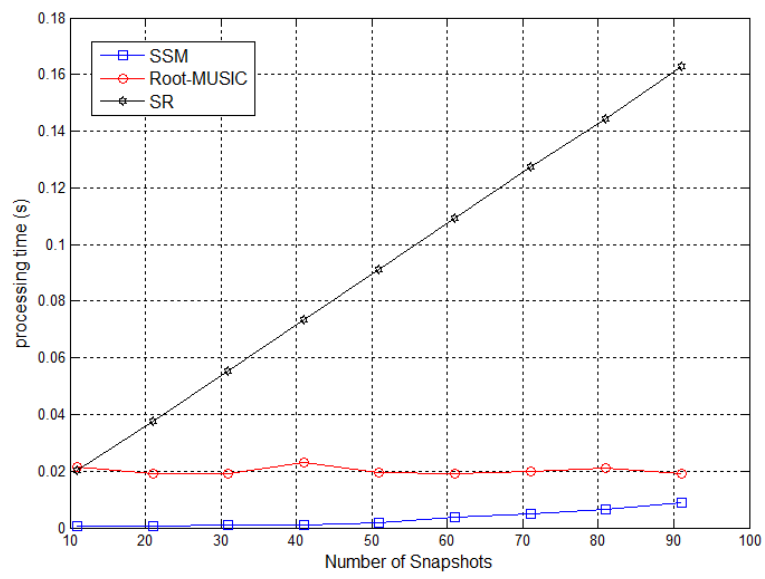

Fig. 9. The processing time versus the number of snapshots

Fig.9 shows the CPU processing time of the three methods at different number of snapshots. The result demonstrates that the SSM is fastest among all, and SR based method turns out to be the slowest. It also show that the computational load of the SR based method grows linearly with the number of snapshots and the computational load of the Root-MUSIC is at least 80 times than that of the SSM. Although the processing time of the SSM increases slightly as the number of snapshots increases, compared with the Root-MUSIC and SR based method, the processing time of the SSM still greatly less than that of the two algorithms.

\section{CONCLUSION}

A novel DOA estimation algorithm, the state space-based method (SSM), is investigated in this paper. The performance of the SSM is compared with Root-MUSIC and SR (sparse representation) based algorithm as well as the CRLB under different SNRs, different number of snapshots and different number of array elements. The SSM outperforms the two method and works well under the case of small snapshots and evenly coherent signal as demonstrated in the numerical experiments. Also, the proposed method is computationally efficient. Although the processing time of the SSM increases as the number of snapshots increases, the processing time increment of the SSM is still very little, comparing with the Root-MUSIC and SR based algorithm, it is negligible. Therefore the proposed method is particularly suited for real time applications over other algorithms.

Finally, it is worth noting that the SSM can be straightforwardly extended to perform the time delay estimation using a finite number of samples. It is also very promising for many other applications such as signal reconstruction, image restoration using small samples.

\section{REFERENCES}

[1] Ye Tian, Xiaoying Sun, "DOA estimation in the presence of unknown non-uniform noise without knowing the number of sources," International Journal of Electronics Letters, vol.44, no.1, pp.108-116, 2014. doi: 10.1080/21681724.2014.966771

[2] P. Charge, Yide Wang, and J. Saillard, "An extended cyclic MUSIC algorithm," IEEE Trans. on Signal Processing, vol.51, no.7, pp.1695-1701, 2003. doi:10.1109/TSP.2003.812834

[3] A. Barabell, "Improving the resolution performance of eigenstructure-based direction-finding algorithms," Proc. of ICASSP, vol.8, pp.336-339,1983. doi: 10.1109/ICASSP.1983.1172124

[4] R. Schmidt, "Multiple emitter location and signal parameter estimation," IEEE Trans. on Antennas and Propagation, vol.34, no.3, pp.276-280, 1986. doi: 10.1109/TAP.1986.1143830

[5] Cheng Qian, Lei Huang, and H. C. So, "Improved unitary Root-MUSIC for DOA estimation based on pseudo-noise resampling," IEEE Signal Processing Letters, vol.21, no.2, pp.140-144, 2014. doi: 10.1109/LSP.2013.2294676

[6] Shu Changgan, Liu Yumin, "An improved forward/backward spatial smoothing Root-MUSIC algorithm based signal decorrelation," Proc. of IEEE WARTIA, pp. 1252-1255, 2014. doi: 10.1109/LSP.2013.2294676

[7] M. D. Zoltowski, S. D. Silverstein, and C. P. Mathews, "Beam-space root-MUSIC for minimum redundancy linear arrays," IEEE Trans. on SignalProcessing,vol.41,no.7,pp.2502-2507,1993. doi: 10.1109/78.224260

[8] Wei Wang, Xianpeng Wang, Xin Li, and Hongru Song, "DOA estimation for monostatic MIMO radar based on unitary root-MUSIC," International Journal of Electronics, vol.100, no.11, pp.1499-1509, 2013. doi :10.1080/00207217.2012.751319

[9] Xiaofei Zhang, Dazhuan Xu, "Improved coherent DOA estimation algorithm for uniform linear arrays," International Journal of Electronics, vol.96, no.2, 213-222, 2009. doi: $10.1080 / 00207210802526810$

[10] A. L. Swindlehurst, T. Kailath, "A performance analysis of subspace-based methods in the presence of model errors, Part I: The MUSIC algorithm," IEEE Trans. on Signal Processing, vol.40, no.7, pp.1758-1774, 1992. doi : 10.1109/78.143447

[11] Schell, S.V, "Performance analysis of the cyclic MUSIC method of direction estimation for cyclostationary signals," IEEE Trans. on Signal Processing, vol.42,no.11, pp.3043 - 3050,1994.doi : 10.1109/78.330364 
[12] Pascal Vallet, Xavier Mestre, and Philippe Loubaton, "Performance analysis of an improved MUSIC DOA estimator," IEEE Trans. on Signal Processing,vol.63,no.23,pp.6407-6422,2015. doi : 10.1109/TSP.2015.2465302

[13] BHASKAR D.RAO, K.V.S.Hari, "Performance analysis of Root-Music," IEEE Transactions on Acoustics, Speech, and Signal Processing, vol.37, no.12, pp.1939-1949, 1989. doi : 10.1109/29.45540

[14] I. F. Gorodnitsky, B. D. Rao, "Sparse signal reconstruction from limited data using FOCUSS: A re-weighted minimum norm algorithm," IEEE Trans. on Signal Processing, vol.45, no.3, pp.600-616, 1997. do : 10.1109/78.558475

[15] D. Malioutov, M. Cetin, and A. S. Willsky, "A sparse signal reconstruction perspective for source localization with sensor arrays," IEEE Trans. on Signal Processing, vol.53, no.8, pp.3010-3022,2005 do : 10.1109/TSP.2005.850882

$\mathrm{Xu} \mathrm{Xu}$, Xiaohan Wei, and Zhongfu Ye, "DOA estimation based on sparse signal recovery utilizing weighted-norm penalty," IEEE signal processing letters, vol.19, no.3, pp.155-158, 2012 doi : 10.1109/LSP.2012.2183592

[16] D. L. Donoho, M. Elad, \& V. Temlyakvo, "Stable recovery of sparse overcomplete representations in the presence of noise," IEEE Trans. Inf Theory, vol.52, pp.6-18, 2006. doi : 10.1109/TIT.2005.860430

[17] BHASKAR D. RAO, "Model based processing of Signals: A state space approach," Proc. of the IEEE, vol.80, no.2, pp.283-309, 1992. do $: 10.1109 / 5.123298$

[18] BHASKAR D. RAO,. "Relationship between matrix pencil and state space based harmonic retrieval methods," IEEE Trans. on Acoust. Speech, Signal processing, vol.38, no.1, pp.177-179,1990. doi: $10.1109 / 29.45568$
[19] S. Y. Kung, K. S. Arun, D. V. Bhaskar Rao, "State space and SVD based approximation methods for the harmonic retrieval problem," Optical Society of America, vol.73, no.12, pp.1799-1811,1983. doi: 10.1364/JOSA.73.001799

[20] B. D. Rao, "Sensitivity considerations in state space model-based harmonic retrieval methods," IEEE Trans. on Acoust., Speech, Signal Processing, vol.37,no.11, pp.1789-1794,1989. doi : 10.1109/29.46567

[21] Hua Chen, "State-space based approximation methods for the harmonic retrieval problem in the presence of known signal poles," Proc. of ICASSP'96, vol.5, pp.2924-2927, 1996. doi : 10.1109/ICASSP.1996.550166

[22] F. Desbouvries, "Unitary Hessenberg and state-space model based methods for the harmonic retrieval problem," Proc. of IEEE Radar, Sonar and navigation, vol.143, no.6, pp.346-348, 1996 doi :10.1049/ip-rsn:19960853

[23] M. Wax, T. Kailath., "Detection of signals by information theoretic criteria," IEEE Trans. Acoust., Speech. Signal Processing, vol.33, no.2, pp. 387-392, 1985. doi : 10.1109/TASSP.1985.1164557

[24] A. Satish, "Maximum likelihood estimation and Cramer-Rao bounds for direction of arrival parameters of a large sensor array,"IEEE Transactions on Antennas and Propagation, vol.44, no.4, pp.478-491,1986. doi :10.1109/8.489299

[25] P. Stoica., "MUSIC, Maximum Likelihood, and Cramer-Rao Bound," IEEE Transactions on Acoustics, Speech, and Signal Processing, vol.37,no.5, pp.720 - 741,1989. doi:10.1109/ICASSP.1988.197097

[26] Joel A. Tropp, Anna C. Gilbert, "Signal recovery from random measurements via orthogonal matching pursuit," IEEE Transactions on Information Theory, vol.53, no.12, pp.4655-4666, 2007. doi:10.1109/TIT.2007.909108 